ISSN-PRINT 1794-9831 / E-ISSN 2322-7028

Vol. $19 \mathrm{~N}^{\circ} 1$ / ene - abr, 2022 / Cúcuta, Colombia.

do $\underline{\text { https://doi.org/10.22463/17949831.3090 }}$

Original

\title{
Enfermería transcultural: puente entre los conocimientos indígenas y científicos sobre dengue
}

\section{Transcultural nursing: bridging indigenous and scientific knowledge on dengue fever Enfermagem transcultural: ponte entre conhecimento indígena e cientifico sobre dengue}

\author{
Nydia Nina Valencia-Jiménez ${ }^{I}$ \\ Concepción Amador-Ahumada ${ }^{2}$ \\ María Camila López-Ortiz ${ }^{3}$
}

\section{Resumen}

Introducción: El dengue es una enfermedad viral documentada por enfoques biomédicos y epidemiológicos, los cuales instituyen la práctica de la enfermería. Sin embargo, hace algunas décadas se plantea la necesidad de crear puentes entre el conocimiento científico y los saberes indígenas para potenciar otras rutas de atención con mayor impacto en la salud humana. Objetivo: Explorar los saberes sobre el dengue que tiene una comunidad indígena, radicada en el municipio de Momil (Córdoba, Colombia). Materiales y métodos: Estudio exploratorio cualitativo con enfoque micro etnográfico, desarrollado entre octubre de 2020 y marzo de 2021. La muestra la conformaron 20 miembros de un cabildo indígena de Momil. Los participantes se seleccionaron por conveniencia, aplicándoseles una entrevista semiestructurada hasta alcanzar el nivel de saturación teórica; estos datos fueron organizados y categorizados bajo la técnica del análisis de contenido de Bardin, empleándose los postulados de Leininger. Resultados: Los indígenas tienen conocimientos atribuidos a la perspectiva universal del cuidado sobre los sitios de cría, síntomas y percepción del riesgo de morir por dengue; sin embargo, presentaron deficiencias vinculadas con el modo de transmisión y ecología del vector Aedes aegypti. Asimismo, se registra la perspectiva del cuidado diverso en el empleo de plantas medicinales como el matarratón, nin, albahaca y orégano para el control del vector y de los síntomas del dengue; resaltan especialmente la visión de ofrecer el cuidado familiar en las primeras fases de la enfermedad. Conclusión: La enfermería transcultural se enuncia como el puente entre el reconocimiento de este tipo de saberes y los conocimientos científicos; no obstante, los entes gubernamentales han de atender las necesidades indígenas que se constituyen en detonantes de las enfermedades.

Palabras clave: Pueblos indígenas; Enfermería transcultural; Conocimiento; Dengue.

\begin{abstract}
Autor de correspondencia*
1* Desarrollista Familiar. Magister en Educación. Docente Programa de Enfermería de la Universidad de Córdoba. Colombia. Correo: nnvalencia@.correo. unicordoba.edu.co (D) 0000-0003-46052430.

${ }^{2}$ Enfermera. Magister en Enfermería Docente Programa de Enfermería de la Universidad de Córdoba. Colombia. Correo: concepcionamador@correo unicordoba.edu.co (D) 0000-0002-0008$\underline{3374}$.

${ }^{3}$ Estudiante último año del Programa de Enfermería de la Universidad de Córdoba. Colombia. Correo: mlopezortiz35@correo.unicordoba.edu.co (1) 0000-0002-1869-2725.
\end{abstract}

Recibido: 16 julio 2021

Aprobado: 6 diciembre 2021

Para citar este artículo

Valencia-Jimenez NN, Amador-Ahumada C, Lopez-Ortiz MC. Enfermería transcultural: puente entre los conocimientos indígenas y científicos sobre dengue. Rev. cien. cuidad. $2022 ; 19(1): 31-41$. https:// doi.org/10.22463/1794983i.3090

(C) Universidad Francisco de Paula Santander. Este es un artículo bajo la licencia CC-BY-NC-ND them until reaching the level of theoretical saturation, these data were organized and categorized under Bardin's content analysis technique, and were used Leininger's postulates for
Introduction: Dengue is a viral disease documented by biomedical and epidemiological approaches, which institute the practice of nursing. However, a few decades ago the need to to promote the use of new alternatives for health care objetive. Exploring the knowledge of an indigenous community of Momil, Colombia, about dengue. Materials and methods: Qualitative exploratory study with a micro ethnographic approach, developed between October 2020 and March 2021, with 20 members of an indigenous community of Momil. The participants were selected for convenience and it was applied a semi-structured interview to 
the analysis. Results: The participants have knowledge attributed to the universal perspective of care about the breeding sites, symptoms and perception of the risk of dying from dengue; However, they presented deficiencies related to the mode of transmission and ecology of the Aedes aegypti vector. Likewise, the perspective of diverse care is recorded in the use of medicinal plants such as matarratón, Nin, basil and oregano for vector control and dengue symptoms; and especially, the vision of offering family care in the early stages of the disease. Conclusion: Transcultural nursing is the bridge between the recognition of this type of knowledge and scientific knowledge, however, the government must attend to the indigenous needs that may be the cause of illnesses.

Key words: Indigenous peoples; Transcultural nursing; Knowledge; Dengue.

\section{Resumo}

Introdução: A dengue é uma doença viral documentada pela perspectiva biomédica e epidemiológica, que institui a pratica de enfermagem. Entretanto, faz algumas décadas planteia-se a necessidade de criar pontes entre o conhecimento cientifico e o saber empírico indígena para fortalecer outras guias de atendimento com maior impacto na saúde humana. Objetivo: explorar o saber sobre a dengue que tem uma comunidade indígena, radicada no município de Momil (Córdoba, Colômbia). Materiais e métodos: Estudo exploratório qualitativo, micro etnográfico, desenvolvido entre outubro de 2020 e março de 2021 . A amostra foi integrada por 20 membros do grupo indígena de Momil. Os participantes selecionaram-se por conveniência, aplicando uma entrevista semiestruturada até conseguir o nível de saturação teórica; esses dados foram organizados e categorizados pela técnica de analise de conteúdo de Bardin, empregando-se a teoria de Leininger. Resultados: Os indígenas tem conhecimentos atribuídos à pespetiva universal do cuidado sobre os sítios de criação, sintomas e percepções do risco de morrer por dengue; entretanto, presentaram deficiências vinculadas com o mecanismo de transmissão e ecologia do vector Aedes aegypti. Além disso, registou-se a perspectiva do cuidado diverso no uso de plantas medicinais conhecidas como mata-rato, nin, manjericão e orégano para o controle do vector e os sintomas da dengue; destacando especialmente, a visão de oferecer o cuidado familiar nas primeiras fases da doença. Conclusão: A enfermagem transcultural é enunciada como a ponte entre o reconhecimento desse tipo de saberes e os conhecimentos científicos; entretanto, os entes governamentais precisam atender as necessidades dos indígenas que constituem detonantes das doenças.

Palavras-chave: Cultura indígena; Enfermagem transcultural; Conhecimento; Dengue.

\section{Introducción}

El dengue es una enfermedad viral de vieja data que impacta la salud humana y amenaza cerca de la mitad de la población mundial, estimándose que existen entre 100 y 400 millones de infecciones principalmente en países de Asia y América Latina (1). El virus se trasmite por la picadura del mosquito hembra del género Aedes aegypti, el cual tiene la capacidad de reproducirse en entornos familiares (2) y afectar a cualquier persona, sin importar su grupo etario, etnia o género. Asimismo, el potencial de reproducción y propagación del vector se vincula a la prestación de servicios públicos inadecuados (3), urbanización acelerada (4), in- adecuadas prácticas preventivas (5) y en las regiones tropicales y subtropicales (6).

En Colombia, coexisten los micro y macro factores que estimulan la emergencia y reemergencia del dengue, datos sustentados por los casos que informa el Ministerio de Salud y Protección Social (Minsalud), entidad que para la semana epidemiológica 40 del año 2020, notificó aproximadamente 70.577 eventos por dengue, lo cual superó los límites esperados para esta etapa (7). Con ello, la apuesta preventiva del dengue involucra, por una parte, la implementación de planes con sistemas de alerta temprana, vigilancia epidemiológica, entomológica y ambiental, laboratorios, notificación 


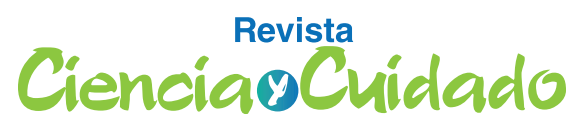

Scientific Journal of Nursing

obligatoria; y por la otra, intervenciones sanitarias sostenibles fundamentadas en la promoción de la educación en salud de las personas, familias y comunidades (8).

En este sentido, la exploración de los saberes, prácticas preventivas y mecanismos para controlar el dengue en comunidades indígenas es un tema de interés para la salud pública, porque estas familias habitan en zonas de alto riesgo como quedó evidenciado con la actual pandemia por COVID-19 por el Minsalud (9), cuando esta institución advierte que los grupos étnicos del país se encuentran localizados en zonas endémicas para dengue y zika, lo cual aumenta la probabilidad de morir por la posible co infección de enfermedades transmitidas por vectores y el SARS-CoV-2, haciendo visible la necesidad de acompañar con mayor efectividad a estas comunidades.

Se debe precisar, que el punto de quiebre en el adecuado acompañamiento del sector salud para combatir el dengue, lo constituye la diversidad de formas de aprender de las comunidades y la necesidad de homogeneizar las formas de enseñanza que limitan el desarrollo de capacidades humanas desde lo que el sujeto cree o aspira (10). Este aspecto es vital en el proceso de promoción y mantenimiento de la salud, al marcar una diferencia significativa entre las comunidades indígenas, porque sus formas de aprendizaje se enuncian desde la visión de lo colectivo, el vínculo con el contexto y la preeminencia del sistema cultural, y en particular por la transmisión de saberes de generación en generación. Al respecto, Leininger propone una discusión en torno al cuidado de la salud desde un enfoque transcultural, reconociendo que las especificidades de este se derivan de lo que cada persona requiere para superar las enfermedades (11).

El abordaje de las problemáticas de salud desde la transculturalidad invita a reconocer las necesidades particulares y los territorios que las producen, de ahí que el personal de la salud está llamado a re-conocer y co-construir las formas individuales y colectivas del cuidado, para promover mejores procesos de intervenciones sanitarias (12). Sin embargo, cuando se está ante el riesgo de extinción de las formas naturales de comprender enfermedades como el dengue, se enfrentan desafíos importantes cuando se pone en duda los saberes y prácticas empleadas por los indígenas que podrían ser la respuesta esperada para erradicar este antiguo pero
Enfermería transcultural: puente entre los conocimientos indígenas y científicos sobre dengue

actual virus (13).

Desde esta perspectiva, el Departamento de Córdoba, ubicado en la costa Caribe de Colombia, se preocupa por presentar diversas alternativas para erradicar el vector transmisor del dengue, por tratarse de una región endémica con un registro de casos importantes que, en 2020 se manifestó en 1.318 casos (14); de igual manera, en el municipio de Momil, escenario de estudio, se presentó un aumento de los casos por dengue nueve veces mayor al del año inmediatamente anterior (15).

Momil, está ubicado en la subregión del bajo Sinú, caracterizándose por ser uno de los municipios del Departamento de Córdoba que ha sido cuna de las más antiguas culturas de América y que aún mantienen vigente gran parte de sus tradiciones culturales. No obstante, el proceso de aculturación se produce con fuerza, por tratarse de una comunidad semiurbana que constantemente intercambia manifestaciones y expresiones con otras comunidades, razones que invitan a realizar estudios cualitativos para sistematizar sus saberes y aportar herramientas a los planes de cuidado que redunden en beneficio de la salud integral de los miembros de esta comunidad.

Se debe subrayar que en este primer acercamiento, se trazó como meta develar los saberes de la comunidad sobre los diferentes aspectos en el proceso de la enfermedad como: la reproducción del vector, modo de trasmisión de la enfermedad, sintomatología y manejo adecuado, debido a que el reconocimiento de estos elementos reducirá la posibilidad de enfermar o morir a causa del dengue.

Hay que mencionar además que los indígenas son sujetos activos en el desarrollo de planes de cuidado; asimismo, es necesario fortalecer los fundamentos teóricos sobre esta enfermedad en los estudios de enfermería que contribuyan con el diseño e implementación de planes de atención, ajustados a las necesidades de cuidado de los pueblos indígenas; al mismo tiempo hay que reducir la brecha que existe entre el desconocimiento desde la perspectiva de la enfermería sobre los cuidados específicos de estas personas, con el objeto de contribuir a la mejoría y prolongación de su bienestar.

\section{Objetivo}




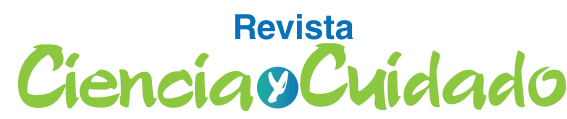

Scientific Journal of Nursing
Nydia Nina Valencia-Jiménez, Concepción Amador-Ahumada, María Camila López-Ortiz
Explorar los saberes sobre el dengue que tiene una comunidad indígena, radicada en el municipio de Momil (Córdoba, Colombia).

\section{Materiales y métodos}

Es un estudio exploratorio cualitativo, por cuanto buscó explorar en profundidad los saberes que posee la comunidad indígena sobre el dengue. Además, se adoptó el enfoque micro etnográfico. La investigación se desarrolló en el periodo comprendido entre octubre de 2020 y el mes de marzo de 2021 en el municipio de Momil, Córdoba, Colombia. Durante este tiempo se procedió a contactar a los líderes indígenas para obtener el aval de acceso a la comunidad y se diseñaron las estrategias para el trabajo de campo; posterior a estas acciones, se llevó a cabo la inmersión en la comunidad mediante en donde se identificaron los informantes clave, las familias participantes y los lugares específicos para aplicar la guía de observación e iniciar el proceso de obtención de los datos.

Los sujetos de estudio fueron indígenas que pertenecen a un cabildo de Momil, quienes fueron seleccionados de manera voluntaria, teniendo en cuenta los siguientes criterios de inclusión: personas que forman parte del cabildo indígena y que se encuentren registradas en su base de datos, mayor o igual a 45 años de edad, oriundos y radicados en el municipio. Entre los criterios de exclusión se tomó en cuenta: persona menor de 45 años, quienes no estén en completo uso de sus facultades mentales, personas no afiliadas al cabildo indígena o afiliadas que habiten en otro municipio, así como aquellos indígenas que se encontraban de forma transitoria en el municipio. El número de participantes se determinó por el nivel de saturación teórica; es decir, las investigadoras suspendieron el reclutamiento de miembros para la muestra, una vez que los datos no ofrecían nuevos elementos para la investigación, estimándose la saturación teórica de las categorías, dando como resultado una muestra de 20 participantes.

En el contexto investigativo, se describe a Momil como un municipio del Departamento de Córdoba con una extensión de 15.550 hectáreas, 17 metros sobre el nivel del mar, clima promedio de $26.1^{\circ} \mathrm{C}$, con un registro de población indígena de 644 personas que estaban inscritos en el cabildo seleccionado para el presente estudio con un total de 586 miembros quienes viven en el casco urbano del municipio (15).

Con respecto a los criterios de selección utilizados para determinar los informantes claves se empleó el método no probabilístico por conveniencia, destacando los principios de pertinencia, adecuación, convivencia, oportunidad y disponibilidad. Seguidamente se realizó la entrevista, elaborada por las investigadoras y revisada por un panel de expertos, quienes refinaron las preguntas acordes a la población objeto de investigación. La aplicación de la entrevista osciló entre 40 y 60 minutos; posteriormente se trascribieron los datos que se obtuvieron a un documento Word con la finalidad de escoger los de mayor relevancia y así ejecutar la fase relacionada con los resultados en función de las mejores características.

Conviene indicar que los criterios de rigor metodológico utilizado para evaluar la calidad científica de la investigación se rigieron según lo establecido por Guba y Lincoln (16) como son: credibilidad, trasferencia, dependencia y confirmabilidad. Por otra parte, se determinó que el proceso de análisis se basa en la técnica de análisis de contenido de Bardin (17), conformado por diferentes fases que facilitan el ordenamiento, la categorización y la definición de las unidades de análisis, mediante los siguientes pasos: fase I o pre análisis, que consiste en la selección de las narrativas de los participantes que se someterían al propósito central del estudio. A continuación se presenta el corpus de análisis aplicando los criterios de exhaustividad (todos los protocolos de recolección de datos se seleccionaron como parte del proceso); de representatividad (se eligió una parte del material representativo para realizar un análisis previo); de homogeneidad (cada texto seleccionado se adaptó a los criterios de selección); y, pertinencia (los textos eran adecuados al propósito del análisis respectivo)

En la fase II: exploración del material, el cual una vez organizado, posteriormente se categoriza. En esta fase se efectuó un plan de cómo hacer el análisis a partir de las operaciones de codificación y sistema de categorías a emplear. Asimismo, los datos primarios se transformaron en unidades que permitieron describir en detalle el contenido analizado, teniendo en cuenta las unidades de registro a partir de ejes temáticos y unidades de contexto; seguidamente se precisaron los significados de las unidades registradas y se aplicaron algunas reglas 
Cienciaø Cuidado

Scientific Journal of Nursing

de: recuento o enumeración, de presencia o ausencia de elementos, frecuencia ponderada e intensidad.

La fase final o proceso de categorización se efectuó a partir de los procedimientos de categorización puros "a priori" con categorías establecidas y derivadas de la revisión de antecedentes y de las bases teóricas. A continuación, una vez transcritas las entrevistas se procedió a leer comprensivamente en varias oportunidades el texto, determinando los atributos categoriales de: homogeneidad, exhaustividad, exclusividad, replicabilidad y pertinencia. Posteriormente, se realizó una matriz de codificación y se ubicaron los códigos in vivo y códigos identificados en la fase anterior. Al mismo tiempo se asociaron las semejanzas en colores y se separaron las diferencias, permitiendo ubicar en primera instancia las subcategorías y la fusión de varias dando como resultado la categoría central.

Hay que mencionar que la investigación de la cual se derivan los resultados del presente artículo, fue sometida a evaluación ante el Comité de ética de la Facultad de Ciencias de la Salud de la Universidad de Córdoba, Colombia. Este comité se aseguró de que los riesgos potenciales que se pudieran derivar del estudio contaran con mecanismos necesarios para su control.

Por otra parte, las investigadoras se acogieron a la Resolución 8430 de 1993, expedida por el Ministerio de Salud y Protección Social en Colombia, cuyo propósito se fundamenta en salvaguardar la identidad del participante, razón por la cual la información se presenta de manera codificada y alfanumérica. Cabe mencionar que cada uno de los entrevistados, firmó con antelación el consentimiento informado, en donde se daba la posibilidad de retirarse del proceso investigativo en el momento que lo consideraran.

\section{Resultados}

Los participantes de la investigación pertenecen a una comunidad indígena del Departamento de Córdoba, ubicada geográficamente en una posición apta para la prevalencia de enfermedades que se trasmiten por vectores como el dengue. Los sujetos de estudio provienen de un entorno con fuertes raíces culturales, con evolución constante por el vínculo permanente con otras comunidades foráneas.
Enfermería transcultural: puente entre los conocimientos indígenas y científicos sobre dengue
Las viviendas como parte de su cultura, se caracterizan por estar construidas con materiales como bahareque, barro y pajas, las cuales por sus largos periodo de existencia sufren un visible deterioro, que potencia sitios de crías para los vectores. La prestación de los servicios públicos es deficiente, aspecto vinculante con la práctica de almacenamiento de agua constante, acumulación de basuras, creación de pozos sépticos (muchos en desuso) y aguas estancadas alrededor de las casas.

Conviene señalar que los participantes cuentan con edades entre 45 y 73 años, predominando un alto porcentaje de mujeres, quienes son amas de casa. Además, todos cuentan con afiliación al sistema se salud vigente bajo la modalidad del régimen subsidiado.

La categoría central del estudio: "Yo conozco cosas por ahí (...)" se abordó a partir de la identificación de subcategorías como: "mosquito flaquito y puntiagudo", "en aguas se cría el mosquito" y "muchas dolencias que te llevan a la muerte", de donde se deduce que corresponden a los saberes de la comunidad acerca del dengue.

Los hallazgos demuestran que un grupo representativo de indígenas reconocen que el dengue es una enfermedad transmitida por vectores, como quedó consignado en sus observaciones:

\section{“(...) Dengue una enfermedad producida por un mos- quito lánguido y con pinticas" “(...) Lo produce un mosquito flaquitico”. (E17) “(...) Por un mosquito grande que tiene las patas blan- cas". (E2)}

Sin embargo, se encontraron registros que catalogan respuestas ambiguas por parte de los indígenas entrevistados, que son erróneas y algunos afirman no conocerlo, como se muestra en los siguientes relatos:

“(...) Dicen que es por el mosquito o el agua sucia”.

“(...) Uff (...) bueno es que yo digo que un mosquito nada más puede infectar a una sola persona, porque dicen que el mosquito pica y se llenó, de ahí no puede picar más, eso dicen". (E20)

“(...) La verdad, no sé cuál es el mosquito ese”. (E7) "(...) Es un mosquito largo, grande, ¿no? Y se forma del parasito que está en las aguas estancadas". (E10) 


\section{CienciànCuidado}

Scientific Journal of Nursing

“(...) Es producida por un parásito que trasmite el mosquito”. (E14)

En este orden de ideas, se percibe que, los entrevistados reconocen diferentes puntos de vista acertados, sobre el lugar específico donde se cría el vector; especialmente señalan como espacio principal para su reproducción los recipientes que almacenan agua por un periodo de tiempo prologado, tal como se refleja en las siguientes líneas:

“(...) Los recipientes que almacenan agua porque ahi es donde él se incuba". (E5)

“(...) Donde hay bastantes aguas estancadas, eso, produce el dengue. Mucha humedad". (E6)

“(...) En aguas estancadas por lo que tenemos que tratar de no acumular esas aguas”. (E11)

“(...) En aguas limpias también son muy propensos a la reproducción”. (E14)

Es pertinente mencionar que, además de reconocer los diferentes síntomas, los participantes conocen la evolución desfavorable que se puede presentar en los cuadros clínicos, particularmente aquellas personas que padecieron dengue o algunos de sus familiares. En pocos casos, se pudo evidenciar la diferenciación conceptual entre dengue clásico y dengue hemorrágico.

"(...) Fiebre, que te produce dolor de cabeza, que te produce malestares en el cuerpo, hemorragias”. (E16) "Dolor en el cuerpo, sin fuerza, salen unos punticos como un brotecito, parece que fuera como una roséola, pero fue del mismo dengue. El dengue les baja las plaquetas". (E20)

"Dolencia en el cuerpo, fiebre alta, eh (...) sangrado si es el caso de dengue hemorrágico”. (E14)

"Del dengue clásico viene el hemorrágico. Entonces hay que evitarlo para (...) para que a uno no le pase nada”. (E9)

"Puede producir la muerte". (E8)

Se resalta en esta subcategoría las expresiones originales de los participantes para referirse a la magnitud de los síntomas del dengue:

“(...) Lo único que me decía era que se estaba acabando y se estaba acabando que se estaba muriendo con un dolor de cabeza, y no sabía de mundo me decía él".
Nydia Nina Valencia-Jiménez, Concepción Amador-Ahumada, María Camila López-Ortiz
“(...) Nos dio dengue a los que habitamos aquí en la casa: nos dio una fiebre alta, dolían todas las articulaciones, se hincha uno todito, más que el brote ese”.

“(...) Un sobrino, casi se muere, duró un mes en UCI. No, no lo dejaban ver, tenía hemorragia”. (E6)

Los anteriores hallazgos dan cuenta de los saberes indígenas enunciados desde una mirada universal de los cuidados, puesto que están construidos en las trayectorias vitales de los entrevistados que, aunque insuficientes, podrían registrarse como aspectos comunes en los diferentes grupos humanos. No obstante, Leininger hace la salvedad, que paralelo a la universalidad en el ejercicio del cuidar existe el cuidado diverso, haciendo referencia a aquellas percepciones, prácticas y saberes diferentes adoptadas por las comunidades e influenciadas por la cultura y el entorno.

Las siguientes expresiones expresan los saberes indígenas para el control del vector:

“(...) Se coge, se mete dentro de unas ollas de esas de aluminio, ya viejas y se le echa ahí y se echa aire y se va prendiendo con unas estee (...) leñas secas y ella va cogiendo un humito y se echa la albahaca y ella". (E6)

“...El nin que sirve para el control de plagas y parásitos". (E5)

“(...) Matarratón es una rama que es muy buena para los mosquitos que uno los espanta y los mata por el olor del matarratón, estas tienen un olor que pa los mosquitos para ellos es como si fuera un fulminante".

“(...) Si yo he utilizado, cuando hay mosquito cojo una rama de matarratón y espanto los mosquitos como que se van, como que se intoxican”. (E7)

De igual forma, se resaltan aquellos saberes indígenas acerca del control de los síntomas del dengue:

“(...) Hay una planta que le dicen la quitadolor que es buena". (E2)

“(...) Cuando me dio yo cociné, yo cocinaba el agua y después que cocinaba el agua le echaba las plantas sea el orégano, sea la quitadolor". (E9) 


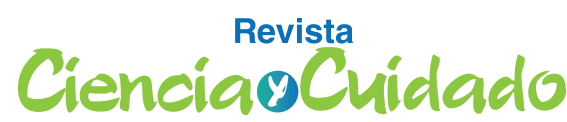

Scientific Journal of Nursing

Otro aspecto a tener en cuenta es la percepción de los indígenas acerca del manejo del dengue en casa, antes de acudir a una atención especializada y la necesidad de conservar sus saberes sin excluir el conocimiento científico como lo enuncian a continuación:

“(...) De pronto yo digo que, de mi arte, de mi experiencia indigena también se le pueden dar unos remedios caseros para para, aguantarlo hasta que llegue a un centro de salud o a un médico, hay que aguantarlo".

“(...) Es bueno enseñarles a los hijos para que no se pierda ese conocimiento, que también puede ser un conocimiento científico. Porque si no se escribe que pierden las memorias vivientes". (E5)

\section{Discusión}

Los miembros del cabildo indígena entrevistados poseen un conocimiento limitado, y en ocasiones, ambiguo e inexistente frente a la transmisión del dengue. Como pudo constatarse, los participantes reconocen en gran medida que esta enfermedad se produce por la picadura de un mosquito, al igual que los datos encontrados por Lliná (18); Giraldo, Álvarez y Parra (19); Riera et al., (20), cuyos sujetos de estudio poseen algún tipo de conocimiento sobre el dengue. Sin embargo, gran parte de las respuestas que se obtuvieron de los indígenas momileros no precisan qué tipo de mosquito en particular transmite el virus del dengue; datos que coinciden con lo manifestado por miembros indígenas de otro resguardo del Departamento de Córdoba, quienes reconocen la causa de transmisión, pero no precisan el tipo de vector o aspectos centrales de su ecología (21).

Otro grupo de respuestas reflejan la confusión de los participantes al creer que el dengue es "producido por un parásito trasmitido por el mosquito" o al limitar el número de picaduras que puede realizar el vector cuando está infectado, siendo similares a los resultados de Atencia y López (22), quienes identifican que sus entrevistados tienen la misma percepción acerca del agente transmisor de la enfermedad; estos hallazgos coinciden con estudiantes indígenas venezolanos, quienes a pesar de poseer ideas claras sobre la transmisión del dengue persisten en hacer hincapié sobre información desactualizada o están desinformados (23).
Enfermería transcultural: puente entre los conocimientos indígenas y científicos sobre dengue
Por otra parte, los indígenas del cabildo "momilero" reconocen la mayoría de los artefactos donde se reproducen y crían los mosquitos del género Aedes aegypti y el vínculo con el almacenamiento de agua, al igual que los indígenas de Tapera en Brasil al dar respuestas en la misma vía (24); en contraste con las respuestas emitidas por el Pueblo shan oriental, en donde se observó desconocimiento acerca de los criadores de mosquitos, vinculado con la ausencia de los servicios de salud y la escasa formación sobre el dengue (25).

Otro de los aspectos abordados en las subcategorías están los síntomas vinculados al dengue reconocidos por los indígenas; al respecto, se encontró que: el aumento de temperatura corporal, cefalea, dolor en las articulaciones y hemorragias constituyeron las principales manifestaciones clínicas reconocidas. Igualmente, los participantes están conscientes sobre la gravedad del dengue, especialmente en aquellos que lo padecieron o vivenciaron de cerca la experiencia, lo cual se constituye en una oportunidad para profundizar acerca del conocimiento sobre el dengue y tener conciencia sobre qué hacer para prevenirlo (25); de igual forma, hay que propiciar actitudes positivas para su control, como lo sugieren en sus conclusiones Benítez, Díaz y Martínez (26), autores que determinaron la existencia del vínculo entre la percepción del riesgo de sufrir dengue con los conocimientos y algunas experiencias con esta arbovirosis.

Definitivamente, los resultados encontrados en la comunidad indígena de Momil (Córdoba, Colombia), conjugan dos asuntos vitales para el análisis: por un lado, la necesidad de documentar el conocimiento de las diferentes culturas, tendientes al uso de este en la planificación de los cuidados como lo postula Leininger; y por otro, la responsabilidad de reflexionar sobre la conexión entre el fomento de prácticas preventivas contra el dengue y la educación transformadora o liberadora.

Al respecto, el modelo de la diversidad y universalidad de los cuidados de Leininger presenta análisis integrales para la prestación del cuidado enfermero bajo la consigna de añadir esfuerzos a la rutina de la enfermería, al ir más allá de los cánones disciplinares. Desde esta perspectiva, se plantea la relación del cuidado transcultural en los procesos de salud frente a la enfermedad y la praxis de la enfermería, tomando como eje central el respeto por la diversidad cultural de cada persona, 


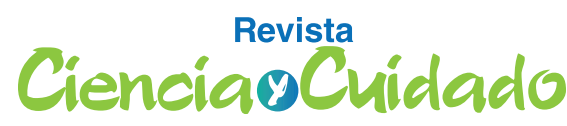

Scientific Journal of Nursing
Nydia Nina Valencia-Jiménez, Concepción Amador-Ahumada, María Camila López-Ortiz familia y comunidad; con ello, la enfermería transcultural promueve a partir de este principio, la autonomía en el ejercicio diario, ofreciendo mayor confort a los pacientes, sin transgredir sus formas particulares de estar, ser y comprender su mundo (27). Al traspasar el universo cultural de las personas, el cuidador descubre y documenta los estilos de vida con influencia directa sobre la toma de decisiones del individuo, y sobre esta base, podrá proponer acciones pertinentes a las necesidades culturales y potenciar el mantenimiento y recuperación de la salud (28)

Por otro lado, existe una tendencia al considerar que las imprecisiones conceptuales sobre las enfermedades son causa directa de las creencias o la vulnerabilidad social del indígena; a partir de esta percepción, el personal de la enfermería construye representaciones sociales que repercuten en la relación enfermeras e indígenas creando en ocasiones algunas barreras en la prestación del servicio. Desde este punto de vista, es necesario poner en práctica capacidades que sirvan para definir e implementar la enfermería transcultural y explorar el mundo indígena con miras a identificar el grado de apropiación de los saberes de estas comunidades, desestimular el aprendizaje de aquellos conocimientos imprecisos y fomentar las prácticas preventivas (29).

Por consiguiente, la reflexión sobre el fomento de prácticas preventivas contra el dengue y la educación transformadora que se configura a partir del análisis acerca de las teorías del aprendizaje, articuladas a las tradiciones culturales y los modelos de mundo, favorecen la construcción de conocimientos para el cuidado de la salud. La vieja dicotomía entre educación tradicional hegemónica y la educación liberadora no solo se produce en escenarios educativos, sino que también se extrapola al campo de la prevención del dengue; al respecto el estudio de Curetti (30), señala que los estudios sobre dengue presentan abordajes que enfatizan en los principios del modelo higienista, la epidemiologia positivista o los determinantes sociales de la salud, con sustento en la causalidad de los eventos de salud y la verticalidad de las intervenciones prácticas del sector salud; al mismo tiempo advierte que para el caso del dengue se enfatiza en el control del vector, las fumigaciones, acciones del Estado y la fiscalización de los estilos de vida de las familias.

Hay que precisar que estos abordajes rígidos sobre el dengue fundamentados en la linealidad del proceso de la salud y la enfermedad desfavorecen la complementariedad teórica que propone la enfermería transcultural, mediante la cual se busca que desde la enfermería se puedan nutrir aquellos saberes óptimos de los grupos indígenas y educar sobre el saber indígena erróneo, pero en escenarios educativos respetuosos y dialógicos. Por ello, se requieren nuevas coordenadas en los planes de cuidados que incluyan miradas integrales para superar el énfasis en el control y eliminación del vector o la exploración de las causas económicas, políticas y laborales (31), porque cada vez es más necesario explorar los saberes, modos de vivir y las interpretaciones que las comunidades realizan sobre sus eventos de salud y analizar sus prácticas sociales tendientes a controlar y prevenir el dengue (32).

De acuerdo con lo mencionado anteriormente, es necesario profundizar sobre la necesidad que tiene el profesional de la enfermería de conocer las diferentes culturas, con la finalidad de brindar cuidados culturalmente congruentes de acuerdo a las creencias, tradiciones y valores que tiene cada grupo cultural, enfatizando en el área educativa, para la promoción del autocuidado respecto a los factores de riesgo de la enfermedad del dengue.

Es importante resaltar que la responsabilidad no solo debería recaer en la enfermería, sino que hay que analizar integralmente el origen histórico, sociocultural, político y educativo factores que permitan propiciar formas y actuaciones estatales y profesionales para combatir el dengue. Es decir, que según se constató las comunidades indígenas aprenden con sus experiencias sobre las enfermedades, conocen fragmentos de la ecología del vector y los principales síntomas del dengue, pero siguen enfermándose con riesgo latente a morir, en gran medida por la desatención del Estado respecto a sus condiciones de vida, déficit en la prestación de los servicios públicos, viviendas inadecuadas y desconocimiento de sus aprendizajes empíricos

\section{Conclusión}

Los indígenas se encuentran ubicados en una zona de alto riesgo para el padecimiento del dengue; paralelo a estas condiciones climáticas y ambientales tienen viviendas aptas a la proliferación del vector, especialmente por los materiales de construcción y el deterioro. Además, existe precariedad en la prestación de los 


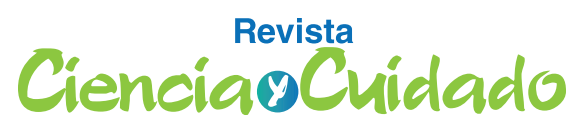

Scientific Journal of Nursing

servicios públicos, factor que favorece las condiciones para la cría de los vectores y la infección por dengue.

Respecto a la problemática expuesta, se constató en los participantes saberes enunciados desde una perspectiva universal coherente con la identificación de sitios de crías, síntomas y percepción del riesgo a morir a causa del dengue; sin embargo, respecto al modo de transmisión de la enfermedad es ambigua entre los entrevistados. Lo anterior, es coherente con un tipo de saber experiencial que se construye con la vivencia y se acumula con la práctica. En el ámbito de lo diverso el estudio permitió confirmar la pervivencia de los saberes indígenas con respecto al manejo de las plantas medicinales para prevenir la propagación del vector y controlar los síntomas. También, se observó en los participantes su perspectiva frente al cuidado de la salud familiar; igualmente, se evidencia la importancia que le otorgan a la conservación de los saberes indígenas, sin desconocer la necesidad de documentarlos frente al conocimiento científico para contribuir de forma más
Enfermería transcultural: puente entre los conocimientos indígenas y científicos sobre dengue acertada en la promoción y mantenimiento de la salud, así como en la adopción de prácticas preventivas contra el dengue.

En definitiva, las bases de la enfermería transcultural invitan a reforzar los estudios previos con diferentes culturas para el mejoramiento en la atención por enfermería, impulsando el fortalecimiento de los cuidados culturales, con la finalidad de percibir a las personas de la misma manera en la que ellos mismos se ven; del mismo modo, se debe promover el empoderamiento de la familia indígena a partir de la educación transformadora como fundamento para el uso de sus costumbres representativas y la diversidad de sus formas de ser, actuar y convivir.

\section{Conflictos de interés}

Los autores declaran no tener ningun conflicto de intereses.

\section{Referencias Bibliográficas}

1. World Health Organization. Dengue and severe dengue. [Internet]. 2020 [citado 2021 Jul 25]. Disponible en: https://www.who.int/es/news-room/fact-sheets/detail/dengue-and-severe-dengue

2. Escalona MG, Izquierdo MR, Romero AIS. Vigilancia y lucha antivectorial en la enfermedad del dengue. Papel del profesional de la salud en la Atención Primaria. Revista urug. enferm. [Internet]. $2017 ; 12$ (1): $269-75$. Disponible en: http://rue.fenf.edu.uy/index.php/rue/article/view/222/215

3. Caraballo A, Chica I, Ocampo M. Comportamiento epidemiológico del Dengue, Chikungunya y Zika. Córdoba-Colombia. 2014-2017. Revista Colombiana Salud Libre. [Internet]. 2017; 12(2): 7-14. Disponible en: https://revistas.unilibre.edu.co/index.php/rcslibre/article/view/1725/1261

4. Aguado-Moquillaza R, Alvites-Junes A, Ceccarelli-Flores J. Determinantes sociales estructurales relacionados con la epidemia de dengue en la ciudad de Ica. Rev. méd. panacea. [Internet]. 2018; (2): 50-55. Disponible en: https://doi.org/10.35563/rmp.v7i2.21

5. Causa R, Ochoa H, Dor A, Rodríguez F, Solís R, Pacheco A. Emerging arboviruses (dengue, chikungunya, and Zika) in Southeastern Mexico: influence of socio-environmental determinants on knowledge and practices. Cadernos de Saúde Pública. [Internet]. 2020; 36(6). Disponible en: https://doi.org/10.1590/0102311X00110519

6. Baldi-Mata G, Hernández-Redondo S, Gómez-López R. Actualización de la fiebre del Dengue. Revista Médica Sinergia. [Internet]. 2020; 5(1). Disponible en: https:/www.medigraphic.com/cgi-bin/new/resumen.cgi?IDARTICULO $=90652$

7. Instituto Nacional de Salud. Boletín Epidemiológico Semanal. [Internet]. 2020 [citado 2021 Jul 30]. Disponible en: https:/www.ins.gov.co/buscador-eventos/BoletinEpidemiologico/2020 Boletin epidemiologico semana_41.pdf

8. Tamayo-Escobar O, García-Olivera T, Escobar-Yéndez N, González-Rubio D, Castro-Peraza O. La reemergencia del dengue: un gran desafío para el sistema sanitario latinoamericano y caribeño en pleno siglo XXI. 
Scientific Journal of Nursing

MEDISAN. [Internet]. 2019; 23(2): 308-24. Disponible en: http://scielo.sld.cu/scielo.php?script=sci arttex$\underline{\text { t\&pid }=\mathrm{S} 1029-30192019000200308 \& \operatorname{lng}=\mathrm{es} \& \mathrm{tlng}=\mathrm{es}}$

9. Ministerio de Salud y Protección Social. Orientaciones para la prevención, detección y manejo de casos de COVID-19 para población étnica en Colombia [Internet]. 2020 [citado $2021 \mathrm{Jul}$ 30]. Disponible en:https:// www.minsalud.gov.co/Ministerio/Institucional/Procesos $\% 20 \mathrm{y} \% 20 \mathrm{procedimientos/TEDS04.pdf}$

10. Valencia Jiménez NN, Carrillo Gullo MA, Ortega Montes JE. Percepción del proceso de alfabetización de jóvenes y adultos indígenas del departamento de Guainía (Colombia): la mirada de sus protagonistas. Invest. Desarro. [Internet]. 2016;24 (1): 118-41. Disponible en: https://www.redalyc.org/pdf/268/26846686006.pdf

11. Tomey AM, Alligood MR. Modelos y teorías de enfermería. Barcelona (España): Elsevier; 2011.

12. Leininger M. Cuidar a los que son de culturas diferentes requiere el conocimiento y las aptitudes de la enfermería transcultural. Cult. cuid. [Internet]. 1999;(6):5-12. Disponible en: https://doi.org/10.14198/cuid.1999.6.01

13. Mattar S, Montero J, Marco GT. La historia del dengue aún no termina. Rev. mvz Córdoba. [Internet]. 2019; 24(2):7177-79. Disponible: http://dx.doi.org/10.21897/rmvz.1597

14. Instituto Nacional de Salud, SIVIGILA. informe de eventos: Dengue, periodo epidemiológico IV Colombia [Internet]. 2020. Disponible en: https://www.ins.gov.co/buscador-eventos/Informesdeevento/DENGUE\%20 PE\%20VI\%202020.pdf

15. Secretaría de Salud de Momil, Instituto Nacional De Salud (INS). Notificación individual por periodos epidemiológicos. UPGD:264640000000. 2021

16. Guba EG, Lincoln YS. Epistemological and methodological bases of naturalistic inquiry. Educ Tech Res Dev. [Internet]. 1982;30(4):233-52. Disponible en: https://www.jstor.org/stable/30219846?seq=1

17. Bardin L. Análisis de contenido. 3 ed. Madrid: Ediciones Akal S.A. 2002

18. Lliná GA. Categorización ambiental para la evaluación de la dinámica espacio-temporal de criaderos artificiales de Aedes aegypti en Clorinda, Argentina (2012-2013) [Tesis de Maestría]. Córdoba, Argentina:CONAE/ UNC; 2020. p.74. Disponible en: https://ig.conae.unc.edu.ar/wp-content/uploads/sites/68/2020/11/Albrieu tesisMAEARTE final.pdf

19. Giraldo-Hurtado TM, Álvarez-Betancur JP, Parra-Henao G. Factores asociados a la infestación domiciliaria por Aedes aegypti en el corregimiento el Manzanillo, municipio de Itagüí (Antioquia) año 2015. Rev Fac Nac Salud Pública. [Internet] 2018;36(1):34-44. Disponible en: https://doi.org/10.17533/udea.rfnsp.v36n1a05

20. Riera AL, De Salas FG, Betancourt CC, Rodríguez RC, De Perales AD. Estrategias de acciones de comunicación y movilización social sobre el dengue en Santa Elena de Uairen del municipio Gran Sabana, estado Bolívar. Bol. Mal. Salud Amb. [Internet]. 2019; 59(2): 90-97. Disponible en: http://iaes.edu.ve/iaespro/ojs/ index.php/bmsa/article/viewFile/72/45

21. Gaviria JP, Humánez ME. Conocimientos y prácticas culturales sobre Dengue en las familias de un cabildo indígena del departamento de Córdoba [Trabajo de grado]. Montería: Universidad de Córdoba; 2020. p.91. Disponible en: https://repositorio.unicordoba.edu.co/bitstream/handle/ucordoba/3425/GaviriaCarvajalJessicaPaola-HumanezPaterninaMaryElen.pdf? sequence=1\&isAllowed $=\mathrm{y}$

22. Atencia AC, Espitia RM. Conocimientos y prácticas ancestrales en la prevención y control del dengue en una comunidad indígena Embera Katío del departamento de Córdoba [Trabajo de grado]. Montería: Universidad de Córdoba; 2021. https://repositorio.unicordoba.edu.co/xmlui/handle/ucordoba/4270

23. Tapia FJ, Villasmíl RC, Moronta R, Romero YM, Parra E, Atencio R, Bracho A. Conocimientos previos sobre transmisión y prevención de enfermedades en comunidades indígenas. Investig. Ensino Ciênc. [Internet]. 2020; 25 (3): 22-40. Disponible en: http://dx.doi.org/10.22600/1518-8795.ienci2020v25n3p22

24. Machado RH, Montenegro F, Malta D, Carlos Henrique CC, Pessoa VE, Araújo LV, Oliveira TC, de Góes LP. 
Scientific Journal of Nursing

Dengue fever and Aedes aegypti in indigenous Brazilians: seroprevalencia, risk factors, knowledge and practice. Trop Med Int Health. [Internet]. 2018; 23(6): 596-604. Disponible en: https://doi.org/10.1111/tmi.13061

25. Jian-Wei X, Liu H, Ai D, Yu Y, Yu B. The Shan people's health beliefs, knowledge and perceptions of dengue in Eastern Shan Special Region IV, Myanmar. PLoS Negl Trop Dis. [Internet]. 2019; 13(6): e0007498. Disponible en: https://doi.org/10.1371/journal.pntd.0007498

26. Benítez-Díaz L, Díaz-Quijano F, Martínez-Vega R. Experiencia y percepción del riesgo asociados a conocimientos, actitudes y prácticas sobre dengue en Riohacha, Colombia. Ciênc. Saúde Colet. [Internet]. 2020; 25 (3): 1137-146. Disponible en: http://dx.doi.org/10.1590/1413-81232020253.08592018

27. Rodrigues da Silva E, Barros de Alencar E, Alves Dias E, Crispim da Rocha L, Canonici Macario S. Transculturalidade na enfermagem baseada na teoria de Madeleine Leininger. Revista Eletrônica Acervo Saúde. [Internet]. 2021; 13(2): e5561. Disponible en: https://doi.org/10.25248/reas.e5561.2021

28. Hernández Garre JM, de Maya Sánchez B. De las "culturas de cuidado" a los "cuidados culturales". Por una antropología del cuidado transdiciplinar. Revista Nuevas tendencias en antropología. [Internet]. 2020; 11: 62-79. Disponible en: http://www.revistadeantropologia.es/Textos/N11/De\%201as\%20culturas\%20del\%20 cuidado $\% 20 \mathrm{a} \% 2010 \mathrm{o} \% 20$ cuidados $\% 20$ culturales.pdf

29. Pérez-Quintero C, Carrasquilla-Baza D. Relación enfermera-paciente frente a la diversidad cultural. Una mirada en situaciones de cuidado a pacientes indígenas. Index Enferm. [Internet]. 2018;27(4):216-220. Disponible en: http://ciberindex.com/c/ie/e12052

30. Curetti PV. De saberes y prácticas biomédicas en torno a la prevención del Dengue en Rosario (Barrio Tablada, año 2011) [Tesis de Maestría]. Argentina: Universidad Nacional del Rosario; 2018. Disponible en: http:// hdl.handle.net/2133/14302

31. López-García C, Betanzos-Reyes AF, Arenas-Monreal ML, Tenahua-Quitl I, De-Ávila-Arroyo ML. Descripción del problema de dengue con enfoque de la determinación social de la salud en una comunidad: estudio de campo. Instituto Investigaciones Ciencias Salud. [Internet]. 2019; 17(2), 6-15. Disponible en: http://scielo. iics.una.py/pdf/iics/v17n2/1812-9528-iics-17-02-6.pdf

32. Guevara M, Marruffo M, Rattia J. Los saberes comunitarios y el control de dengue. Una experiencia en el Municipio Mario Briceño Iragorry. Espacio Abierto. Cuaderno venezolano de Sociología. [Internet]. 2018;28(1):22752. Disponible en: https://produccioncientificaluz.org/index.php/espacio/article/view/29719/30641 